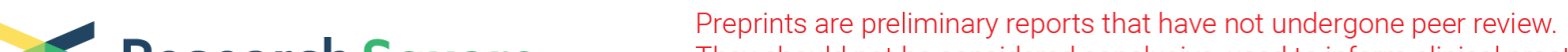

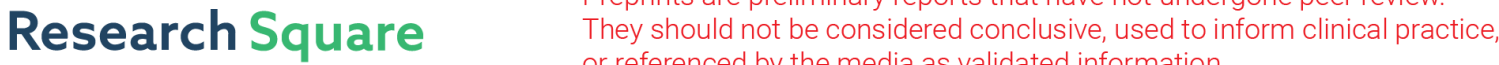 or referenced by the media as validated information.
}

\section{Assessment of Structural Disconnections In Gliomas: Comparison of Indirect And Direct Approaches}

Erica Silvestri

University of Padova https://orcid.org/0000-0002-1853-0777

Umberto Villani

University of Padova https://orcid.org/0000-0002-2016-6943

Manuela Moretto

University of Padova https://orcid.org/0000-0003-4178-1854

Maria Colpo

University of Padova https://orcid.org/0000-0001-8509-8569

Alessandro Salvalaggio

University of Padova https://orcid.org/0000-0002-1273-7566

Mariagiulia Anglani

University of Padova https://orcid.org/0000-0002-3989-4553

Marco Castellaro

University of Padova https://orcid.org/0000-0002-1203-2670

Silvia Facchini

University of Padova

Elena Monai

University of Padova

Domenico D'Avella

University of Padova

\section{Alessandro Della Puppa}

University of Florence https://orcid.org/0000-0001-8058-092X

Diego Cecchin

University of Padova

\section{Maurizio Corbetta}

University of Padova https://orcid.org/0000-0001-8295-3304

Alessandra Bertoldo ( $\nabla$ alessandra.bertoldo@unipd.it )

University of Padova https://orcid.org/0000-0002-6262-6354 
Keywords: Structural disconnection, glioma, diffusion MRI, tractography

Posted Date: December 13th, 2021

DOI: https://doi.org/10.21203/rs.3.rs-1117189/v1

License: (c) (1) This work is licensed under a Creative Commons Attribution 4.0 International License. Read Full License 


\section{Abstract}

Gliomas are amongst the most common primary brain tumours in adults and are often associated with poor prognosis. Understanding the extent of white matter (WM) which is affected outside the tumoral lesion may be of paramount importance to explain cognitive deficits and the clinical progression of the disease. To this end, we explored both direct (i.e., tractography based) and indirect (i.e., atlas based) approaches to quantifying WM structural disconnections in a cohort of 50 high- and low-grade glioma patients. While these methodologies have recently gained popularity in the context of stroke, to our knowledge this is the first time they are applied in patients with brain tumours. More specifically, in this work we present a quantitative comparison of the disconnection maps provided by the two methodologies by applying well known metrics of spatial similarity, extension and correlation. Given the important role the oedematous tissue plays in the physiopathology of tumours, we performed these analyses both by including and excluding it in the definition of the tumoral lesion. This was done to investigate possible differences determined by this choice.

We found that direct and indirect approaches offer two distinct pictures of structural disconnections in patients affected by brain gliomas, presenting key differences in several regions of the brain. Following the outcomes of our analysis, we eventually discuss the strengths and pitfalls of these two approaches when applied in this critical field.

\section{Introduction}

Gliomas constitute the most common malignant primary brain tumours in adults and are associated with significant morbidity and mortality (Ostrom et al. 2014). Gliomas frequently develop from glial cells and, according to the World Health Organization (WHO), they are classified into different histological grades that reflect their malignancy or aggressiveness (Louis et al. 2016, 2021). High-grade gliomas (HGG; grades III and IV) are considered malignant tumours and are treated more aggressively than low-grade gliomas (LGG; grades I and II). Standard treatment for gliomas includes neurosurgical resection followed by concomitant chemotherapy and radiation therapy. In this regard, the major goal in surgical planning is to preserve eloquent brain regions and structural connections while removing most of the tumoral tissue (Ghinda et al. 2018; Castellano et al. 2017; Duffau 2019). Due to the highly infiltrative characteristics of these tumours, the impact of gliomas on the structural connectivity of the brain is complex to assess and is currently an open issue in contemporary neuroscience (Nilsson et al. 2018). Since gliomas commonly extend towards gyri and then spread along the course of white matter (WM) tracts, they can be distinguished not only by their anatomic location, but also by which WM tract they have infiltrated.(Glenn et al. 2017). A richer depiction of the topological alterations caused by the tumour on the structural connectome may lead to a better understanding of the complex network dysfunctions the pathology causes (Sporns 2011; Castellano et al. 2017) and could identify cortical regions and WM bundles to be carefully navigated during surgery (Duffau 2019). Techniques which identify and quantify the extent WM damage beyond the location of a focal lesion have recently been gaining popularity in the field of stroke, as they tend to explain functional alterations better than damage to specific grey matter 
(GM) regions (Griffis et al. 2019; Thiebaut de Schotten, Foulon, and Nachev 2020; Di Vita et al. 2019; Salvalaggio et al. 2020). Translating the use of such techniques in the oncological field requires, however, additional cares as the neoplastic nature of tumours poses some difficult challenges to the investigation of the alteration of WM tracts. Indeed, gliomas do not represent a focal and acute lesion but rather a slowly evolving infiltrative process.

When studying the course of WM pathways through imaging, diffusion MRI (dMRI) is the instrument of choice, as it allows to extract the state and directional information of WM fibres through modelling the random motion of water molecules occurring in the different brain regions. Through dMRI, patterns of WM structural disconnections can be derived exploiting so-called indirect or direct approaches:

- Indirect approaches bypass the need of a subject specific dMRI dataset by projecting the tumoral lesion of a given patient onto a tract-based atlas to detect which WM pathways are most likely to be affected by the pathology (Foulon et al. 2018). Although indirect methods may be more readily accessible, they currently generalize the glioma to be one lesioned region causing homogeneous effects on WM pathways. In this way, the high heterogeneity of the underlying pathological tissues (i.e., oedema, necrosis, tumoral core comprising different cell dimensions and cellularity) and their specific effects on axonal bundles is completely disregarded.

- Direct approaches involve the quantification of the brain connectome through tractography algorithms applied on dMRI data. Such methodologies are known to have their own limitations and pitfalls (Jeurissen et al. 2019) but, contrary to indirect approaches, have the undisputed advantage of providing subject-specific quantification of WM bundles.

Given the intrinsic difference between direct and indirect approaches, the aim of this study is to propose a quantitative comparison between them. We apply simple but effective image analyses to evaluate benefits and criticalities of both, highlighting points of agreement and divergence in terms of WM disconnection information that can be derived. Moreover, as the oedematous tissue appears to play a crucial role in defining tissutal regions which are subject to inflammation, favourable pathways for tumour spreading, we additionally investigate how patterns of structural disconnections are quantified when the oedematous tissue is included in the definition of pathological lesions.

\section{Materials And Methods}

Forty-four patients suffering from de novo brain tumours have been recruited and acquired at the University Hospital of Padova from July 2017 to March 2021. All procedures were in accordance with the ethical standards of the institutional research committee and with the 1964 Helsinki declaration plus later amendments. All participants provided informed, written consent in accordance with the local University Hospital Institutional Review Board.

Figure 1 shows a comprehensive overview of the analyses introduced in this section. 


\subsection{MRI Acquisition}

Data acquisition was performed with a 3T Siemens Biograph mMR-PET/MR scanner equipped with a 16channels head-neck coil. The multi-shell dMRI protocol comprised a total of 100 diffusion weighted images (DWIs) (TR/TE 5355/104 ms; voxel size 2x2x2 mm3; FOV 220×220 mm²; 68 slices; multiband accelerator factor=2): 10 images at $b=0 \mathrm{~s} / \mathrm{mm}^{2}, 30 \mathrm{DWls}$ at $b$-value $=710 \mathrm{~s} / \mathrm{mm}^{2}$ and $60 \mathrm{DWls}$ at $b$ value $=2855 \mathrm{~s} / \mathrm{mm}^{2}$. This diffusion HARDI protocol is the optimized two shells NODDI protocol as described in (Zhang et al. 2012). Each diffusion direction was acquired with reverse phase encoding directions, i.e., anterior-posterior and posterior-anterior directions, for distortion correction purposes.

In addition, the acquisition protocol included anatomical imaging, which comprised a 3D T2-weighted (T2w) Fluid Attenuated Inversion Recovery (FLAIR) image (TR/TE 5000/395 ms; voxel size 1x1x1 mm; FOV 250x250 $\mathrm{mm}^{2}$ ), two 3D T1-weighted (T1w) magnetization-prepared rapid acquisition gradient echo (MPRAGE, TR/TE 2400/3.2 ms; voxel size 1x1x1 mm³ FOV 256x256 mm²; 160 slices) acquired both before and after contrast agent injection and a T2w image (TR/TE $3200 / 536 \mathrm{~ms}$; voxel size $1 \times 1 \times 1 \mathrm{~mm}^{3}$; FOV 256x256 mm²; 160 slices).

\subsection{Tumour Segmentation and Structural Pre-processing}

The anatomical images of each patient were linearly registered to the patient naïve $T 1 \mathrm{w}$ image with the Advanced Normalization Tools (ANTs (Avants et al. 2011), v. 2.0.1). Employing these images, two masks were manually delineated through the ITK-SNAP software (http://www.itksnap.org/) by an expert neuroradiologist with more than five years of experience. The first mask, the $T$, included the tumour core (contrast agent enhancing and non-enhancing regions) and the necrosis, where present. The second mask, the $T+O$, was created by adding the oedema area to the $T$ mask. In addition, each tumour was labelled by the same neuroradiologist as left, right or bilateral according to the location of its core and to the mainly involved hemisphere.

Structural pre-processing was applied to the T1w image of each patient and consisted in bias field correction (N4BiasFieldCorrection (Tustison et al. 2010)), skull-stripping (Multi-Atlas Skull Stripping (Doshi et al. 2013)), tissue segmentation (into GM, WM and cortico-spinal fluid, with the unified segmentation tool (Ashburner and Friston 2005) of SPM12 v. 7771) and diffeomorphic non-linear registration (as implemented in ANTs SyNalgorithm) to the symmetric MNI152 atlas. The last step was performed excluding the $T+O$ area from the computation of the cost function as suggested in (Andersen, Rapcsak, and Beeson 2010).

For each patient, the $T$ and $T+O$ masks were then mapped into the MNI152 exploiting the estimated diffeomorphic non-linear transformations. 
Finally, since direct and indirect disconnection mapping methods differ in the tracking of potential disconnections within subcortical areas, for each patient, we used the AAL3 atlas (Rolls et al. 2020) to segment the following regions: thalamus, caudates, putamen, pallidum, and hippocampus. Such areas were discarded not to bias the subsequent methods' comparison.

\subsection{Disconnection Maps Computation}

\subsubsection{Direct Disconnection Map}

Diffusion MRI Pre-processing: The acquired diffusion weighted volumes were visually inspected to identify and remove those images affected by interslice instabilities (J. D. Tournier, Mori, and Leemans 2011) which were deemed excessively corrupted for subsequent pre-processing techniques to correct. The rest of the pre-processing was executed in its entirety within the MRtrix3 Software (J.-D. Tournier et al. 2019) and was featured by an initial denoising step based on random matrix theory (Veraart, Fieremans, and Novikov 2016), and a subsequent call to the tools TOPUP (Andersson, Skare, and Ashburner 2003) and eddy (Andersson and Sotiropoulos 2016) from the FMRIB Software library (FSL) for $\mathrm{BO}$ inhomogeneity, eddy current and motion joint correction.

T1w segmentation results (including GM, subcortical parcellation, lesion and tumour masks) were registered to the naïve B0 volume using ANTs, by applying an affine transformation previously estimated on the patient's naïve T1w image.

Diffusion tractography specifications: The patient structural connectome reconstruction was performed in its entirety within the MRtrix 3 software. We firstly performed multi-shell multi-tissue spherical deconvolution (Jeurissen et al. 2014) to recover the orientation distribution functions for each voxel. Subsequently, we computed the structural connectome by employing Anatomically Constrained Tractography (R. E. Smith et al. 2012), tracking individual fibres with a second-order Integration over Fiber Orientation Distributions algorithm (J.-D. Tournier, Calamante, and Connelly 2010). Standard streamline termination criteria values were used. The number of generated streamlines for each patient initially amounted to $100 \mathrm{M}$, which were quantitatively reduced to $10 \mathrm{M}$ via the Spherical-deconvolution Informed Filtering of Tractograms framework (R. Smith et al. 2013).

Disconnection Maps Computation: For each patient, following the quantification of the diffusion tractogram, we computed the patterns of structural disconnections by taking the two following steps:

1. We computed the subset of the streamlines in the tractogram that featured an overlap with the tumoral lesion (respectively, with $T$ and, after, with $T+0$ masks).

2. In the dMRI space (i.e., the naïve space of the tractogram), we computed how many altered streamlines (the subset in step 1) were passing through each voxel of the brain. We labelled direct structural disconnection (dSD) maps these voxel-wise frequency maps. 
We used in-house MATLAB (ver. 2020b, The Mathworks, Natick, MA) scripts to perform the creation of the dSD maps.

As further volumetric analyses of dSD maps required their binarization, the definition of a threshold to define significant disconnection was necessary. We defined such threshold with the following procedure:

1. Similarly to the dSD map quantification, we computed for each subject in the dMRI space a voxelwise streamline density map, this time considering the entirety of the tractogram.

2. We brought the individual streamline density maps to the MNI 152 space via the previously estimated diffeomorphic transformations.

3. In the common MNI152 space, we computed the population average of the individual maps, omitting the lesioned ROI on a patient-by patient basis.

4. To balance the presence of a higher number of patients with left tumours, we symmetrised the frequency template by flipping (left-right) the obtained map, summing the flipped and non-flipped maps and dividing by 2 .

5. Using the same transform as in 2 , we projected back the population-averaged streamline density map to the T1w space of each subject. We referred this resulting map as AvgDensity.

6. Finally, we considered the structural disconnection value of voxel with coordinates $(x, y, z)$ as significant if the following criterion was met:

$\operatorname{dSD}(x, y, z) / \operatorname{AvgDensity}(x, y, z)>10 \%$

Additional investigations were performed also using more stringent and permissive thresholds (i.e., $5 \%, 15 \%, 20 \%, 25 \%$ ).

Hence for each patient we obtained a mask of significantly disconnected voxels.

\subsubsection{Indirect Disconnection Maps Computation}

Indirect structural disconnection (iSD) maps were quantified with the BCB Toolkit v. 4.2 (Foulon et al. 2018; Thiebaut de Schotten, Foulon, and Nachev 2020). In choosing which healthy controls tractography atlas to use within the toolbox, we opted for the extended diffusion dataset provided by the toolkit authors, for which the structural connectome of 180 healthy from the Human Connectome Project' 7T controls was quantified (full tractography specification in (Thiebaut de Schotten et al. 2017)).

In summary, using the BCB Toolkit, for each patient, the lesion masks (both $T$ and $T+O$ ) in the MNI152 space were registered to each control naïve space using affıne and diffeomorphic deformations and subsequently used as seed for the tractography in TrackVis (http://trackvis.org). Tractographies from the lesions were transformed in visitation maps, binarized and brought to the MNI152. Finally, the percentage overlap map was computed by summing at each point in the MNI152 space the normalized visitation map of each healthy subject. Hence, in the resulting disconnectome map (i.e., iSD), the value in each 
voxel considers the tracts' interindividual variability and indicate a probability of disconnection from 0 to $100 \%$ for the given lesion.

As no ad-hoc studies are available regarding the recommended use of iSD maps in tumours, we set the probabilistic threshold to 0.5 as in the software defaults. Additional investigations were performed also using more stringent and permissive thresholds (i.e., 0.7 and 0.3 respectively).

\subsection{Metrics of Comparison}

Having obtained a total of four different SD maps (i.e., two for each methodology, different in terms of the employed input mask: $T$ and $T+O$ ), we wanted to compare them both intra-methodology (i.e., same approach, $\mathrm{T}$ versus $\mathrm{T}+\mathrm{O}$ maps) and inter-methodology (i.e., same lesion mask, direct versus indirect maps).

The framework we employed for their comparison had three simple metrics, useful to quantify different similarity features:

- The difference in volume ( $\Delta V o$ ): : This metric allowed us to quantify the difference in the extension of alteration which is detected by two disconnection maps.

- The Dice Similarity index (Dice): The Sørensen-Dice similarity index is a well-known metric of comparison between digital images and is defined by the following formula:

$$
\text { Dice }=\frac{2(A \cap B)}{|A|+|B|}
$$

where $A, B$ are the two binary matrices for which the similarity needs to be tested. The Dice index quantifies how similar the shape of structural alterations is between two different approaches.

- The Correlation at the intersection (Corn): We computed the Pearson correlation in a region of interest defined by the intersection of the two matrices in exam. Unlike the Dice index which evaluates only the similarity between shapes, the correlation analysis considers where the hotspots of alteration are in the two SD maps, and measures their spatial agreement.

While the $\Delta \mathrm{Vo}$ / and Dice metrics were calculated using the binarized disconnection maps, the Corr index was computed using the thresholded SD maps.

The Dice and the $\Delta \mathrm{Vo} /$ indexes were computed both considering the entire disconnection maps and subdividing them into their ipsilateral and contralateral components (bilateral tumours were excluded from this last analysis). 
To summarise the obtained indexes, we computed the median value and the $25^{\text {th }} / 75^{\text {th }}$ percentiles across all subjects for each metric of comparison.

\subsection{Statistical Analysis}

To test for statistically significant differences in the $\Delta V o l$, both considering intra-method and inter-method results at the whole brain or considering only the ipsilateral or contralateral hemisphere, a Wilcoxon rank sum test (significance level $a=0.05$ ) was employed.

To assess whether there was a linear relationship between the comparison metrics and the extension of the input mask, we performed a correlation analysis (Spearman Correlation, significance level $\mathrm{a}=0.05$ ) between the three indexes (i.e., $\triangle \mathrm{Vol}$, Dice and Corr) and the volume of the input mask, separately for $T$ and $T+O$ masks. To test the sensitivity of the comparison metrics to the set of thresholds used for the $\mathrm{dSD} / \mathrm{iSD}$ maps, we defined their normalized range of variation $(\mathrm{nRV})$ as

$$
\mathrm{nRV}(\mathrm{k})=100 * \frac{\left|\max \left(\mathrm{k}\left(t_{i S D}, t_{d S D}\right)\right)-\min \left(\mathrm{k}\left(t_{i S D}, t_{d S D}\right)\right)\right|}{\max \left(\mathrm{k}\left(t_{i S D}, t_{d S D}\right)\right)}
$$

where $\left.\mathrm{k}\left(t_{i S D}, t_{d S D}\right)\right)$ is the median value across the dataset of the comparison metric between two SD maps, given the indirect $\left(t_{i S D}\right)$ and direct ${ }^{\left(t_{S D}\right)}$ thresholds. The investigated thresholds values were $t_{d S D}[5 \%, 10 \%, 15 \%, 20 \%, 25 \%]$ and $t_{i S D}[0.3,0.5,0.7]$.

\section{Results}

Patient's main demographic and clinical information are summarized in Table 1. A detailed description of the single patient characteristics is provided in the Supplementary Table 1. Overall, according to 2021 World Health Organization classification of tumours of the central nervous system (Louis et al. 2021), 33 patients had a glioblastoma, 1 had an astrocytoma, 3 had a glioneuronal and neuronal tumours, 1 had an oligodendroglioma, 1 had a primary diffuse large B-cell lymphoma, 2 had other types of brain tumour (1 intracranial mesenchymal tumour and 1 non otherwise specified tumour) and 3 had an unclassifiable brain tumour, as they deceased shortly before the surgery or did not underwent neurosurgery. The extent of the $T+O$ mask ranged between 5.7 and $191.6 \mathrm{~cm}^{3}$ (mean value $65.1 \mathrm{~cm}^{3}$, std $50.4 \mathrm{~cm}^{3}$ ), whereas the extent of the T mask between 0.4 and $155.9 \mathrm{~cm}^{3}$ (mean value 46.5 , std $39.1 \mathrm{~cm}^{3}$ ).

Figure 2 shows the frequency maps of the lesions in the patient population. The two reported maps refer to the $T$ (first two rows) and to the $T+O$ masks (second two rows). The distribution is sparse with tumours 
involving predominantly the right frontal and temporal lobes, with a low spatial overlap (maximum value $17.8 \%$ of patients for the $T$ mask, and $22.2 \%$ of patients for the $T+O$ mask).

Figure 3 shows an example of disconnection maps for two representative subjects in our dataset. In panel A we can clearly see that the dSD map is able to detect the displacement of axonal fibres in the brain due to the tumoral mass (most apparent in the axial and coronal views). This effect is not detectable by the indirect approach, which inevitably leads to key differences between methodologies $\left(\Delta V o /\left(\mathrm{iSD}_{\mathrm{T}+0}, \mathrm{dSD}_{\mathrm{T}+0}\right)=-28.05 \mathrm{~cm}^{3} ; \Delta V o /\left(\mathrm{iSD}_{\mathrm{T}}, \mathrm{dSD}_{\mathrm{T}}\right)=-4.8 \mathrm{~cm}^{3} ; \operatorname{Dice}\left(\mathrm{iSD}_{\mathrm{T}+0}, \mathrm{dSD}_{\mathrm{T}+0}\right)=0.51\right.$; $\left.\left.\operatorname{Dice}\left(\mathrm{iSD}_{T}, \mathrm{dSD}_{\mathrm{T}}\right)=0.51 ; \operatorname{Corr}\left(\mathrm{iSD}_{\mathrm{T}+0}, \mathrm{dSD}_{\mathrm{T}+0}\right)=0.45 ; \operatorname{Corr}\left(\mathrm{iSD}_{T}, \mathrm{dSD}_{\mathrm{T}}\right)=0.43\right)\right)$. While the displacement of fibres is less visually appreciable in panel $\mathrm{B}$, differences between approaches are still present $\left(\Delta V o /\left(\mathrm{iSD}_{\mathrm{T}+0}, \mathrm{dSD}_{\mathrm{T}+0}\right)=-21.1 \mathrm{~cm}^{3} ; \Delta V o /\left(\mathrm{iSD}_{\mathrm{T}}, \mathrm{dSD}_{\mathrm{T}}\right)=-4.1 \mathrm{~cm}^{3} ; \operatorname{Dice}\left(\mathrm{iSD}_{\mathrm{T}+0}, \mathrm{dSD}_{\mathrm{T}+0}\right)=0.59\right.$; Dice $\left.\left(\mathrm{iSD}_{\mathrm{T}}, \mathrm{dSD}_{\mathrm{T}}\right)=0.51 ; \operatorname{Cor}\left(\mathrm{iSD}_{\mathrm{T}+0}, \mathrm{dSD}_{\mathrm{T}+0}\right)=0.41 ; \operatorname{Corr}\left(\mathrm{iSD}_{T}, \mathrm{dSD}_{\mathrm{T}}\right)=0.33\right)$ ). Looking at the SD maps intramethodology, i.e., comparing between $\mathrm{T}$ and $\mathrm{T}+0$ maps, the difference is less self-evident and, apart from a few areas where $T+0$ maps show more extensive structural disconnections, we find analogous behaviours and morphological features. While $\Delta V \mathrm{~V} /$ remains high, suggesting the presence of volumetric differences between the maps, this is well reflected by the Dice and Corr metrics being sensibly higher, both in A. $\left(\Delta V o /\left(\mathrm{dSD}_{\mathrm{T}+0}, \mathrm{dSD}_{\mathrm{T}}\right)=25 \mathrm{~cm}^{3} ; \Delta V o /\left(\mathrm{iSD}_{\mathrm{T}+0}, \mathrm{iSD}_{\mathrm{T}}\right)=15.17 \mathrm{~cm}^{3} ; \operatorname{Dice}\left(\mathrm{dSD}_{\mathrm{T}+0}, \mathrm{dSD}_{\mathrm{T}}\right)=0.74\right.$; $\left.\left.\operatorname{Dice}\left(\mathrm{iSD}_{\mathrm{T}+0}, \mathrm{iSD}_{\mathrm{T}}\right)=0.84 ; \operatorname{Corr}\left(\mathrm{dSD}_{\mathrm{T}+0}, \mathrm{dSD}_{\mathrm{T}}\right)=0.79 ; \operatorname{Cor}\left(\mathrm{iSD}_{\mathrm{T}+0}, \mathrm{iSD}_{\mathrm{T}}\right)=0.89\right)\right)$ and in $\mathrm{B}$. $\left(\Delta V o /\left(\mathrm{dSD}_{\mathrm{T}+0}, \mathrm{dSD}_{\mathrm{T}}\right)=39.36 \mathrm{~cm}^{3} ; \Delta V o /\left(\mathrm{iSD}_{\mathrm{T}+0}, \mathrm{iSD}_{\mathrm{T}}\right)=22.40 \mathrm{~cm}^{3} ; \operatorname{Dice}\left(\mathrm{dSD}_{\mathrm{T}+0}, \mathrm{dSD}_{\mathrm{T}}\right)=0.75 ;\right.$ $\left.\left.\operatorname{Dice}\left(\mathrm{iSD}_{\mathrm{T}+0}, \mathrm{iSD}_{\mathrm{T}}\right)=0.85 ; \operatorname{Corr}\left(\mathrm{dSD}_{\mathrm{T}+0}, \mathrm{dSD}_{\mathrm{T}}\right)=0.92 ; \operatorname{Corr}\left(\mathrm{iSD}_{\mathrm{T}+0,} \mathrm{iSD}_{\mathrm{T}}\right)=0.86\right)\right)$.

Generalizing Fig. 3 results, Table 2 shows the metrics employed for the comparison in the entire dataset and reports their median and their $25^{\text {th }} / 75^{\text {th }}$ percentiles. Comparing intra-method results, as expected, we obtained a positive $\Delta \mathrm{Vo}$ / which means that the volume of disconnection increased when $T+O$ is used as input mask (mainly in the whole brain analysis and in the ipsilateral hemisphere). In addition, we obtained a good agreement between the compared maps highlighted both by Dice and by Corr indices, suggesting that in both cases analogous spatial patterns of disconnection were detected. On the other hand, the inter-method comparison revealed that: 1 ) the detected volume of disconnection is significantly lower at the whole-brain and ipsilateral level for the indirect method when the $T+O$ mask is used ( $\mathrm{p}$-val respectively of 0.005 at whole brain, 0.0008 at ipsilateral level; 2) overall there is a decrease in the agreement of the disconnection location (whole brain, ipsilateral and contralateral) with a median Dice value of $0.57 ; 3$ ) there is a poor agreement of the disconnection pattern with a median Corr value of 0.45 and 0.39 respectively for the $T$ and $T+O$ input masks.

No significant linear relationship was found between the extension of the input map and the similarity of dSD/iSD maps.

Fig. 4 shows the sensitivity of the median of the computed metrics across the dataset to a set of possible thresholds. Considering inter-methodology comparisons (green-stars and red triangles in the figure), we found the $V o l$ index to be the most variable across the tested thresholds $\left(n R V_{T+0}(\Delta V o l)=280.34 \%\right.$, 
$\left.n R V_{\mathrm{T}}(\Delta V o l)=210.34 \%\right)$. Morphology similarities were more consistent $\left(\mathrm{nRV}_{\mathrm{T}+0}(\right.$ Dice $)=35.58 \%$, $n R V_{T}($ Dice $\left.)=29.53 \%\right)$, as well as the agreement of hotspot locations $\left(\mathrm{nRV}_{\mathrm{T}+0}(\right.$ Corr $)=26.58 \%, \mathrm{nRV}_{\mathrm{T}}($ Corr $)=$ $31.32 \%$ ). Shifting to intra-methodology comparisons (blue circles and purple squares in the figure), as expected, we found a more stable situation. Again, volumetric measures were the most variable across thresholds $\left(\mathrm{nRV}_{\mathrm{T}+0}(\Delta V O)=52 \%, \mathrm{nRV}_{\mathrm{T}}(\Delta V O)=48.24 \%\right)$, and an even higher consistency was found considering the Dice $\left(\mathrm{nRV}_{\mathrm{T}+0}(\right.$ Dice $)=4.79 \%, \mathrm{nRV}_{\mathrm{T}}($ Dice $\left.)=2.45 \%\right)$, and Corr indexes $\left(\mathrm{nRV}_{\mathrm{T}+0}(\right.$ Corr $)=4.79 \%$, $\mathrm{nRV}_{\mathrm{T}}($ Corr $\left.)=3.91 \%\right)$.

\section{Discussion And Conclusions}

Throughout the recent years, several warnings have been raised against the use of indirect approaches to investigate the disruption of structural connectivity in neoplastic pathologies, as physio-pathological phenomena such as tissue displacement (Clark et al. 2003) or transneuronal degeneration (Fornito, Zalesky, and Breakspear 2015) are not considered by atlas-based methodologies. On the other hand, diffusion tractography techniques may enable the quantification of these effects in individual patients. The framework of analysis we introduced in this article allows to systematically compare patterns of WM disconnections and quantify differences between methodologies. Thus, we here investigated the difference between direct and indirect approaches to quantify patterns of WM disconnections in subjects suffering from brain tumours.

It is important to emphasize that both approaches compute a disconnection map, i.e., a volume that contains the fibres that intersect the tumour core or the tumour core + oedema. However, while the indirect approach defines volumes that contain fibres from a healthy normative atlas going through the regions of interest (tumour, or tumour + oedema) (Foulon et al. 2018), the direct approach defines volumes where the direct measured tractography differs from the pseudo-normative values tractography directly at the patient level.

We compared disconnection maps obtained with either approach using their overall volume, the Dice index to evaluate their shape similarities, and finally a correlation analysis aimed to investigate if hotspots of disconnections were identified accordingly. We carried out these analyses both using the tumour core alone or also including the region of surrounding oedema. The latter allows to define the impact of oedematous tissue in the computation of SD maps using either direct or indirect methods.

Interestingly, the volume of disconnection was significantly higher when using the direct vs. the indirect method, especially when the oedema was also considered. This increase occurred particularly in the hemisphere ipsilateral to the tumour, where fibres are most likely to intersect the tumoral lesion.

Contralateral fibres, on the other hand, are less likely to intersect the tumoral lesion and the detection of their alterations did not appear to be influenced as much by the oedema. In general, the inclusion of the oedema appeared to drastically alter the extension of ipsilateral patterns of disconnections but did not severely impact the detection of contralateral alteration. 
For what concerns the spatial similarity between the investigated maps, the Dice coefficient revealed good intra-methodology agreement, with both direct/indirect maps featuring high values when testing the difference in terms of inclusion of the oedema in the tumoral lesion. When comparing direct and indirect methodologies however, the Dice coefficient showed lower values indicating a substantial mismatch between the structural disconnection patterns. Such differences remained consistent even when we considered ipsilateral and contralateral hemispheres separately.

Dice values for inter- and intra- methodology maps were well reflected by the Corr index, which revealed whether the localization of hotspots of alterations was concordant between structural disconnection maps. As expected, intra-methodology correlations were strong, with hotspots of alterations having the same spatial location. When we investigated inter-methodology relationships, however, such correspondence was lost, regardless of whether the oedema was included or excluded in the analysis.

Overall, these differences lead us to think that the impact of the inclusion of the oedema in the tumoral lesion has a minor impact than varying between direct/indirect approaches. However, the effect of such choice is still non-negligible and produces sensible changes in the structural disconnection patterns that can be observed.

We initially hypothesized that the size of the tumour would play a pivotal role in the similarity between the patterns of disconnections. However, no significant linear relationship was found between the studied metrics and the tumoral lesion extension (see Supplementary Materials for details of the analysis).

The sensitivity analysis to the thresholds for indirect and direct disconnection maps revealed that volumetric indexes are moderately dependent on such choice, potentially altering the interpretation of its results.

We are aware there are some limitations to our work. First and foremost, the structural disconnection maps must be thresholded to make any inference regarding the volume and shape of the detected disconnections. This choice is non-trivial, and while there are some literature indications (although not specifically in tumour cases) for indirect methodologies (Thiebaut de Schotten et al. 2015), thresholding tractograms is an open question in the field of dMRI (Yeh et al. 2021). Volumetric measures of disconnection were significantly impacted by threshold choice, whereas the Dice and Corr indexes were less affected. These observations lead us to raise a warning on the use of volumetric measures when relating structural disconnection features to other quantities of clinical interest. As a second limitation, there are differences in place between the streamline reconstruction of the tract-based atlas of the BCB toolkit, and of the patients belonging to our dataset. While using the same tractography algorithm in both methodologies would indeed eliminate the variability in results due to the different tracking, the purpose of this study was to use the indirect disconnection tools "as is", and to compare such results with stateof-the-art tracking techniques. Moreover, "voxellizing" the streamlines to the disconnection maps arguably reduces the variability due to diffusion orientation sampling and streamline shapes. Thus, we believe this discretization step highlights in the final SD maps those differences between the two approaches which are mainly due to dominant physio-pathological effects rather than fibre reconstruction intricacies. As a 
last note, few studies have shown that the presence of a tumoral lesion may cause Wallerian degeneration to take place in the surrounding area and at distance from its location (Saksena et al. 2013; Lahrmann et al. 2005; Sawlani et al. 1997). This physio-pathological process represents the inflammatory response of the nervous system to an axonal injury and may significantly impair the permeability of axons in those regions (Pierpaoli et al. 2001), limiting the possibility for tractography algorithms to robustly reconstruct the associated streamlines. Depending on the severity of the degeneration, such WM regions may not be visible to direct approaches, which would consequently fail to detect sites of ongoing pathological alteration.

In conclusion, with our work we presented evidence that direct and indirect approaches offer two different pictures of structural disconnections in patients affected by brain tumours. Given these differences, we advise that whenever mass displacement effects appear to be present, direct methodologies should be preferred as they are better suited to account for these morphological and pathological variations. Nevertheless, assessing structural disconnection maps' predictive value of biological/cognitive progression of glioma patients is vital to addressing the clinical relevance of these techniques. Thus, further studies are needed to answer these unmet demands.

\section{Declarations}

\section{Funding}

No funding was received to assist with the preparation of this manuscript.

\section{Financial and Non-financial interest}

The authors declare they have no financial and non-financial interests.

\section{Conflicts of interest/Competing interests}

The authors declare no conflict of interest that are relevant to the context of the present work.

\section{Availability of data and material}

Data used in the present study can be accessed via request to the corresponding author.

\section{Code availability}

The codes and processed data that support the conclusions of this research work can be accessed via request to the corresponding author.

\section{Authors' contributions}

E.S., U.V., M.M. and A.B. designed the study and performed the analysis, interpretation of the data, and the drafting of the article. M.Colpo performed part of the tractography analysis. A.S. and M.Corbetta 
contributed to the interpretation of the data. E.S. and D.C. collected the data. E.S., U.V., M.M., M.Colpo, A.S., M.A., M.Castellaro, S.F., E.M., D.D.A., A.D.P., D.C., M.Corbetta, A.B. reviewed the article and approved its final version.

\section{Ethics approval}

This study was approved by the Ethics Committee of University Hospital of Padova (No. 2771P prot:0065859/12).

\section{Consent to participate}

All participants were provided with written informed consent, which they signed prior to any experimental procedures.

\section{Consent to publication}

All authors discussed the study, read the manuscript, and approved its submission to your journal. The manuscript has not been published previously, and it is not under consideration for publication elsewhere.

\section{References}

1. Andersen, Sarah M, Steven Z Rapcsak, and Pélagie M Beeson. 2010. "Cost Function Masking during Normalization of Brains with Focal Lesions: Still a Necessity?" Neurolmage 53 (1): 78-84.

2. Andersson, Jesper L.R., and Stamatios N. Sotiropoulos. 2016. "An Integrated Approach to Correction for Off-Resonance Effects and Subject Movement in Diffusion MR Imaging." Neurolmage 125: 1063-78. https://doi.org/10.1016/j.neuroimage.2015.10.019.

3. Andersson, Jesper L R, Stefan Skare, and John Ashburner. 2003. "How to Correct Susceptibility Distortions in Spin-Echo Echo-Planar Images: Application to Diffusion Tensor Imaging." Neurolmage 20 (2): 870-88. https://doi.org/10.1016/S1053-8119(03)00336-7.

4. Ashburner, John, and Karl J Friston. 2005. “Unified Segmentation." Neurolmage 26 (3): 839-51. https://doi.org/10.1016/j.neuroimage.2005.02.018.

5. Avants, Brian B., Nicholas J. Tustison, Gang Song, Philip A. Cook, Arno Klein, and James C. Gee. 2011. "A Reproducible Evaluation of ANTs Similarity Metric Performance in Brain Image Registration." Neurolmage 54 (3): 2033-44. https://doi.org/10.1016/j.neuroimage.2010.09.025.

6. Castellano, Antonella, Sara Cirillo, Lorenzo Bello, Marco Riva, and Andrea Falini. 2017. "Functional MRI for Surgery of Gliomas." Current Treatment Options in Neurology 19 (10).

7. Clark, Chris A., Thomas R. Barrick, Mary M. Murphy, and B. Anthony Bell. 2003. "White Matter Fiber Tracking in Patients with Space-Occupying Lesions of the Brain: A New Technique for Neurosurgical Planning?" Neurolmage. https://doi.org/10.1016/j.neuroimage.2003.07.022. 
8. Doshi, Jimit, Guray Erus, Yangming Ou, Bilwaj Gaonkar, and Christos Davatzikos. 2013. "Multi-Atlas Skull-Stripping." Academic Radiology 20 (12): 1566-76.

9. Duffau, Hugues. 2019. "Surgery for Malignant Brain Gliomas: Fluorescence-Guided Resection or Functional-Based Resection?" Frontiers in Surgery 6: 21. https://doi.org/10.3389/fsurg.2019.00021.

10. Fornito, Alex, Andrew Zalesky, and Michael Breakspear. 2015. "The Connectomics of Brain Disorders." Nature Reviews Neuroscience. https://doi.org/10.1038/nrn3901.

11. Foulon, C., L. Cerliani, S. Kinkingnéhun, R. Levy, C. Rosso, M. Urbanski, E. Volle, and M. Thiebaut de Schotten. 2018. "Advanced Lesion Symptom Mapping Analyses and Implementation as BCBtoolkit." GigaScience 7 (3). https://doi.org/10.1093/gigascience/giy004.

12. Ghinda, Diana C., Jin Song Wu, Niall W. Duncan, and Georg Northoff. 2018. "How Much Is EnoughCan Resting State FMRI Provide a Demarcation for Neurosurgical Resection in Glioma?" Neuroscience and Biobehavioral Reviews 84 (December 2017): 245-61. https://doi.org/10.1016/j.neubiorev.2017.11.019.

13. Glenn, Chad, Andrew K Conner, Meherzad Rahimi, Robert G Briggs, Cordell Baker, and Michael Sughrue. 2017. "Common Disconnections in Glioma Surgery: An Anatomic Description." Cureus 9 (10). https://doi.org/10.7759/cureus.1778.

14. Griffis, Joseph C., Nicholas V. Metcalf, Maurizio Corbetta, and Gordon L. Shulman. 2019. “Structural Disconnections Explain Brain Network Dysfunction after Stroke." Cell Reports. https://doi.org/10.1016/j.celrep.2019.07.100.

15. Jeurissen, Ben, Maxime Descoteaux, Susumu Mori, and Alexander Leemans. 2019. "Diffusion MRI Fiber Tractography of the Brain." NMR in Biomedicine. https://doi.org/10.1002/nbm.3785.

16. Jeurissen, Ben, Jacques-Donald Tournier, Thijs Dhollander, Alan Connelly, and Jan Sijbers. 2014. "Multi-Tissue Constrained Spherical Deconvolution for Improved Analysis of Multi-Shell Diffusion MRI Data." Neurolmage 103 (December): 411-26. https://doi.org/10.1016/j.neuroimage.2014.07.061.

17. Lahrmann, Heinz, Barbara Horvath-Mechtler, Peter Hitzenberger, Stefan Oberndorfer, Walter Struhal, and Wolfgang Grisold. 2005. "Pyramidal Tract Degeneration in Astrocytoma." Journal of NeuroOncology 72 (3): 271-72. https://doi.org/10.1007/s11060-004-2342-6.

18. Louis, David N., Arie Perry, Guido Reifenberger, Andreas von Deimling, Dominique Figarella-Branger, Webster K. Cavenee, Hiroko Ohgaki, Otmar D. Wiestler, Paul Kleihues, and David W. Ellison. 2016. "The 2016 World Health Organization Classification of Tumors of the Central Nervous System: A Summary." Acta Neuropathologica 131 (6): 803-20. https://doi.org/10.1007/s00401-016-1545-1.

19. Louis, David N, Arie Perry, Pieter Wesseling, Daniel J Brat, lan A Cree, Dominique Figarella-Branger, Cynthia Hawkins, et al. 2021. "The 2021 WHO Classification of Tumors of the Central Nervous System: A Summary." Neuro-Oncology 23 (8): 1231-51. https://doi.org/10.1093/neuonc/noab106.

20. Nilsson, Markus, Elisabet Englund, Filip Szczepankiewicz, Danielle van Westen, and Pia C. Sundgren. 2018. "Imaging Brain Tumour Microstructure." Neurolmage. https://doi.org/10.1016/j.neuroimage.2018.04.075. 
21. Ostrom, Quinn T, Luc Bauchet, Faith G Davis, Isabelle Deltour, James L Fisher, Chelsea Eastman Langer, Melike Pekmezci, et al. 2014. 'The Epidemiology of Glioma in Adults: A 'State of the Science' Review." Neuro-Oncology 16 (7): 896-913. https://doi.org/10.1093/neuonc/nou087.

22. Pierpaoli, Carlo, Alan Barnett, Sinisa Pajevic, Robert Chen, La Roy Penix, Anette Virta, and Peter Basser. 2001. "Water Diffusion Changes in Wallerian Degeneration and Their Dependence on White Matter Architecture." Neurolmage 13 (6): 1174-85. https://doi.org/10.1006/nimg.2001.0765.

23. Rolls, Edmund T, Chu-Chung Huang, Ching-Po Lin, Jianfeng Feng, and Marc Joliot. 2020. "Automated Anatomical Labelling Atlas 3." Neurolmage 206: 116189. https://doi.org/https://doi.org/10.1016/j.neuroimage.2019.116189.

24. Saksena, Sona, Rajan Jain, Lonni Schultz, Quan Jiang, Hamid Soltanian-Zadeh, Lisa Scarpace, Mark Rosenblum, Tom Mikkelsen, and Mohammad Reza Nazem-Zadeh. 2013. "The Corpus Callosum Wallerian Degeneration in the Unilateral Brain Tumors: Evaluation with Diffusion Tensor Imaging (DTI)." Journal of Clinical and Diagnostic Research 7 (2): 320-25. https://doi.org/10.7860/JCDR/2013/4491.2757.

25. Salvalaggio, Alessandro, Michele De Filippo De Grazia, Marco Zorzi, Michel Thiebaut de Schotten, and Maurizio Corbetta. 2020. "Post-Stroke Deficit Prediction from Lesion and Indirect Structural and Functional Disconnection." Brain: A Journal of Neurology 143 (7): 2173-88. https://doi.org/10.1093/brain/awaa156.

26. Sawlani, Vijay, Rakesh K. Gupta, Manoj K. Singh, and Anoop Kohli. 1997. "MRI Demonstration of Wallerian Degeneration in Various Intracranial Lesions and Its Clinical Implications." Journal of the Neurological Sciences 146 (2): 103-8. https://doi.org/10.1016/S0022-510X(96)00299-7.

27. Smith, Robert E, Jacques-Donald Tournier, Fernando Calamante, and Alan Connelly. 2012.

"Anatomically-Constrained Tractography: Improved Diffusion MRI Streamlines Tractography through Effective Use of Anatomical Information." Neurolmage 62 (3): 1924-38.

https://doi.org/10.1016/j.neuroimage.2012.06.005.

28. Smith, Robert, Jacques-Donald Tournier, Fernando Calamante, and Alan Connelly. 2013. "SIFT: Spherical-Deconvolution Informed Filtering of Tractograms." Neurolmage 67 (February): 298-312. https://doi.org/10.1016/j.neuroimage.2012.11.049.

29. Sporns, Olaf. 2011. "The Human Connectome: A Complex Network." Annals of the New York Academy of Sciences. https://doi.org/10.1111/j.1749-6632.2010.05888.x.

30. Thiebaut de Schotten, Michel, Flavio Dell'Acqua, P Ratiu, A Leslie, H Howells, E Cabanis, M T IbaZizen, et al. 2015. "From Phineas Gage and Monsieur Leborgne to H.M.: Revisiting Disconnection Syndromes." Cerebral Cortex 25 (12): 4812-27. https://doi.org/10.1093/cercor/bhv173.

31. Thiebaut de Schotten, Michel, Chris Foulon, and Parashkev Nachev. 2020. "Brain Disconnections Link Structural Connectivity with Function and Behaviour." Nature Communications 11 (1): 5094. https://doi.org/10.1038/s41467-020-18920-9.

32. Thiebaut de Schotten, Michel, Marika Urbanski, Benedicte Batrancourt, Richard Levy, Bruno Dubois, Leonardo Cerliani, and Emmanuelle Volle. 2017. "Rostro-Caudal Architecture of the Frontal Lobes in 
Humans." Cerebral Cortex 27 (8): 4033-47. https://doi.org/10.1093/cercor/bhw215.

33. Tournier, Jacques-Donald, Fernando Calamante, and Alan Connelly. 2010. "Improved Probabilistic Streamlines Tractography by 2nd Order Integration over Fibre Orientation Distributions." Proceedings of the International Society for Magnetic Resonance in Medicine, 1670.

34. Tournier, Jacques-Donald, Robert Smith, David Raffelt, Rami Tabbara, Thijs Dhollander, Maximilian Pietsch, Daan Christiaens, Ben Jeurissen, Chun-Hung Yeh, and Alan Connelly. 2019. "MRtrix3: A Fast, Flexible and Open Software Framework for Medical Image Processing and Visualisation." Neurolmage 202 (November): 116137. https://doi.org/10.1016/j.neuroimage.2019.116137.

35. Tournier, Jacques Donald, Susumu Mori, and Alexander Leemans. 2011. "Diffusion Tensor Imaging and Beyond." Magnetic Resonance in Medicine. https://doi.org/10.1002/mrm.22924.

36. Tustison, Nicholas J, Brian B Avants, Philip A Cook, Yuanjie Zheng, Alexander Egan, Paul A Yushkevich, and James C Gee. 2010. "N4ITK: Improved N3 Bias Correction." IEEE Transactions on Medical Imaging 29 (6): 1310-20. https://doi.org/10.1109/TMI.2010.2046908.

37. Veraart, Jelle, Els Fieremans, and Dmitry S Novikov. 2016. “Diffusion MRI Noise Mapping Using Random Matrix Theory." Magnetic Resonance in Medicine 76 (5): 1582-93. https://doi.org/10.1002/mrm.26059.

38. Vita, Antonella Di, Liana Palermo, Maddalena Boccia, and Cecilia Guariglia. 2019. "Topological Map of the Body in Post-Stroke Patients: Lesional and Hodological Aspects." Neuropsychology 33 (4): 499-507. https://doi.org/10.1037/neu0000536.

39. Yeh, Chun Hung, Derek K. Jones, Xiaoyun Liang, Maxime Descoteaux, and Alan Connelly. 2021. "Mapping Structural Connectivity Using Diffusion MRI: Challenges and Opportunities." Journal of Magnetic Resonance Imaging. https://doi.org/10.1002/jmri.27188.

40. Zhang, Hui, Torben Schneider, Claudia A. Wheeler-Kingshott, and Daniel C. Alexander. 2012. "NODDI: Practical in Vivo Neurite Orientation Dispersion and Density Imaging of the Human Brain." Neurolmage 61 (4): 1000-1016. https://doi.org/10.1016/j.neuroimage.2012.03.072.

\section{Tables}

Table 1: Patient's main demographic and clinical information for the cohort of subjects included in this study. n.a. $=$ not available. 


\section{Gender}

\begin{tabular}{ll}
\hline Female $(\mathrm{n})$ & 20 \\
\hline Male $(\mathrm{n})$ & 24 \\
\hline Tumour Histology & \\
\hline Astrocytoma $(\mathrm{n})$ & 1 \\
\hline Glioblastoma $(\mathrm{n})$ & 33 \\
\hline Glioneuronal and neuronal tumours $(\mathrm{n})$ & 3 \\
\hline Oligodendroglioma (n) & 1 \\
\hline Primary diffuse large B-cell lymphoma (n) & 1 \\
\hline Other (n) & 2 \\
\hline n.a (n) & 3
\end{tabular}

Tumour Grade

\begin{tabular}{cc}
\hline Low $(\mathrm{n})$ & 5 \\
\hline High $(\mathrm{n})$ & 36 \\
\hline n.a. $(\mathrm{n})$ & 3 \\
\hline IDH-1 Mutation Status & \\
\hline Wild Type (n) & 29 \\
\hline Mutated (n) & 5 \\
\hline n.a. $(\mathrm{n})$ & 10 \\
\hline
\end{tabular}

Tumour Site

\begin{tabular}{lc}
\hline Left $(n)$ & 22 \\
\hline Right $(n)$ & 17 \\
\hline Bilateral $(n)$ & 5 \\
\hline
\end{tabular}

Table 2: median values (with $25^{\text {th }}$ and $75^{\text {th }}$ percentile in squared brackets) for the similarity metrics computed across the patient cohort. Each column represents a comparison between two specific SD maps (e.g., the first column is the intra-methodology comparison between the SD maps generated with the $T$ and $T+O$ lesion masks). For further specificity, we additionally divided the analysis of the Dice and $\Delta \mathrm{Vol}$ indexes in the hemispheres ipsilateral and contralateral to the presence of the tumour. 


\begin{tabular}{|c|c|c|c|c|c|}
\hline & & $\begin{array}{l}\text { COMPARISOI } \\
\text { METHOD }\end{array}$ & DF INTRA- & $\begin{array}{l}\text { COMPARISOI } \\
\text { METHOD }\end{array}$ & F INTER- \\
\hline & & DISCONNEC7 & NMAPS & DISCONNECT & NMAPS \\
\hline & & INDIRECT $_{T+0}$ & DIRECT $_{T+0}$ & INDIRECT $_{\mathrm{T}+0}$ & INDIRECT $_{T}$ \\
\hline & & INDIRECT $_{T}$ & DIRECT $_{T}$ & DIRECT $_{T+0}$ & DIRECT $_{T}$ \\
\hline EXTENSION & $\Delta \mathrm{VOL}$ & 9.17 & 27.4 & -25.1 & -0.97 \\
\hline & $\left(\mathrm{cm}^{3}\right)$ & [0.08 29.4] & [0.11 61.3] & {$[-46.2-12.3]$} & {$\left[\begin{array}{lll}-21.4 & 8.1\end{array}\right]$} \\
\hline & $\Delta$ VOL IPSILATERAL & 5.55 & 20.2 & -21.2 & -0.43 \\
\hline & $\left(\mathrm{cm}^{3}\right)$ & [0.07 19.6] & [0.05 50.3] & {$[-33.3-10.3]$} & {$\left[\begin{array}{lll}-16 & 6.3\end{array}\right]$} \\
\hline & $\triangle \mathrm{VOL}$ & 2.31 & 2.16 & -1.76 & -0.18 \\
\hline & $\left(\mathrm{cm}^{3}\right)$ & [0 7.63] & [0 9.83] & {$\left[\begin{array}{lll}-11.1 & 0.62\end{array}\right]$} & $\begin{array}{l}{[-4.64} \\
1.58]\end{array}$ \\
\hline SPATIAL & DICE & 0.9 & 0.83 & 0.57 & 0.46 \\
\hline & & {$\left[\begin{array}{ll}0.62 & 1\end{array}\right]$} & {$[0.391]$} & [0.52 0.59] & {$\left[\begin{array}{lll}0.37 & 0.57\end{array}\right]$} \\
\hline & DICE IPSILATERAL & 0.9 & 0.85 & 0.57 & 0.46 \\
\hline & & {$\left[\begin{array}{lll}0.70 & 1\end{array}\right]$} & {$\left[\begin{array}{lll}0.46 & 1\end{array}\right]$} & {$\left[\begin{array}{lll}0.51 & 0.6\end{array}\right]$} & [0.35 0.59$]$ \\
\hline & DICE & 0.86 & 0.88 & 0.53 & 0.44 \\
\hline & & {$\left[\begin{array}{lll}0.42 & 1\end{array}\right]$} & {$\left[\begin{array}{lll}0.17 & 1\end{array}\right]$} & {$\left[\begin{array}{lll}0.35 & 0.61]\end{array}\right]$} & {$\left[\begin{array}{ll}0 & 0.59\end{array}\right]$} \\
\hline STRUCTURE & CORRELATION in the & 0.91 & 0.87 & 0.45 & 0.39 \\
\hline & & {$[0.741]$} & {$\left[\begin{array}{ll}0.72 & 1\end{array}\right]$} & {$\left[\begin{array}{lll}0.39 & 0.50\end{array}\right]$} & [0.32 0.48$]$ \\
\hline
\end{tabular}

\section{Figures}




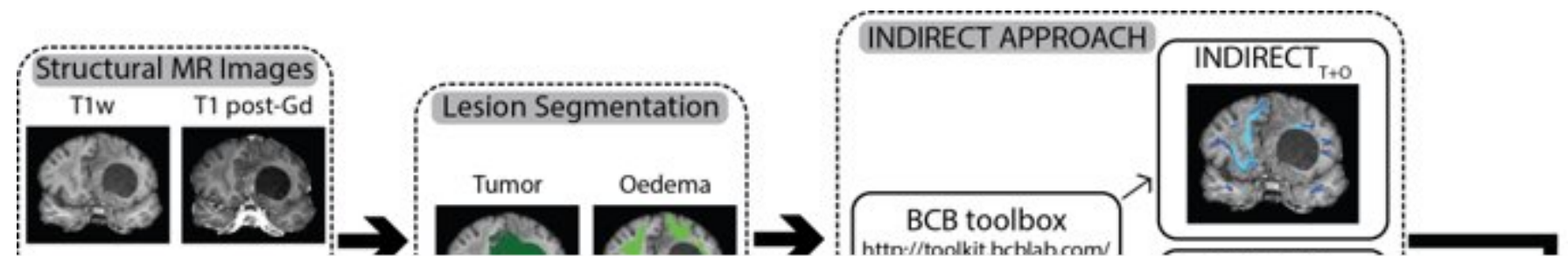

\section{Figure 1}

Visualisation of the processing pipeline which led to the computation of the four SD maps (upper row: indirect approach; lower row: direct approach) which were eventually compared.

\section{Figure 2}

Frequency maps of tumoral lesions in our cohort of patients. In the two upper rows, the lesion was composed by the tumoral core (both enhancing and non-enhancing, and necrotic regions). In the two lower rows, the tumoral lesion mask was composed by the tumour core and the oedematous tissue.

\section{Figure 3}

Lesion segmentation (dark green = oedema, green=tumour core), iSD (blue/light-blue) and dSD (red/yellow) maps overlayed on the T1w image for two representative subjects from our cohorts of patients. In the SD maps, the lighter the colour is, the higher the probability/severity of WM disconnection.

\section{Figure 4}


Sensitivity to iSD/dSD thresholds for the similarity metrics in inter- and intra-methodology comparisons. Individual points of the same shape and colour (e.g., green stars, representing the inter-methodology comparison of $\mathrm{T}+\mathrm{O}$ maps) are the median values across the dataset for the given index, computed with a different pair of $\mathrm{dSD} / \mathrm{iSD}$ thresholds.

\section{Supplementary Files}

This is a list of supplementary files associated with this preprint. Click to download.

- SupplementarySilvestriVillani.docx 